\title{
Calidad de vida y autodeterminación en Centros de Educación Ordinaria: Estudio de casos múltiples
}

\section{Quality of life and self-determination in Regular Educational Centers: Multiple case study}

\author{
Jesús Miguel Muñoz-Cantero, Luisa Losada-Puente \\ Dpto. Filosofía y Métodos de Investigación en Educación. Universidad de A Coruña (España)
}

\begin{abstract}
Resumen
Este estudio analiza la autodeterminación y la calidad de vida de diez adolescentes con discapacidad intelectual escolarizados en Centros Educativos Ordinarios. Se selecciona la metodología de estudio de caso descriptivo-interpretativa. Los resultados son positivos en cinco de las siete dimensiones de la calidad de vida; mejorable en el caso de la autodeterminación, donde se observa una baja percepción de la capacidad de estos jóvenes para actuar de forma autodeterminada. Destaca la cercana relación entre mayor calidad de vida y autodeterminación, y otras variables (entorno educativo, actitud del profesorado, influencia de la relación familia-escuela en las oportunidades de los jóvenes).

Palabras clave: calidad de vida, autodeterminación, discapacidad, estudiantes con necesidades educativas especiales.
\end{abstract}

\begin{abstract}
This study examines self-determination and quality of life of ten adolescents with intellectual or developmental disabilities schooled in Regular Educational Centers. A case study methodology, descriptive-interpretative, is selected. The results are positive in five of the seven dimensions of quality of life; improved in the case of self-determinations, where a low perception of the ability of these teens to act self-determined is observed. It stresses the close relationship between higher quality of life and self-determination, and other variables (educational environment, thachers' attitudes, influence of family-school relationship in opportunities for teens). Keywords quality of life, self determination, disabilites, special needs students.
\end{abstract}

\section{Introducción}

Desde hace más de tres décadas, la proliferación y difusión de estudios sobre calidad de vida ha permitido ampliar el conocimiento acerca del significado de este concepto y su importancia en el campo de la discapacidad. Si bien en su origen ha sido asociada a multitud de situaciones deseables y positivas de las personas, hoy en día sabemos que su significado va más allá de aspectos como la felicidad, el bienestar o el buen estado de salud, extendiéndose cada vez más hacia una perspectiva comprehensiva e integradora; esto es, un constructo que guía las actuaciones de los servicios que buscan mejorar las condiciones de vida y la satisfacción de las personas, así como un medio para lograr una mayor participación, equidad y satisfacción con la propia vida (Claes, Van Hove, Van Loon, Vandevelde y Schalock, 2010).

En el terreno educativo, la calidad de vida se erige como eje prioritario de las intervenciones en pro de la inclusión educativa (Schalock, Gardner y Bradley, 2009). El movimiento inclusivo llega a España en 1994, cuando se celebra la Conferencia Mundial sobre Necesidades Educativas Especiales (Salamanca) donde se debate sobre la necesidad de aportar mayor claridad en los mensajes sobre integración/inclusión promovidos desde las políticas educativas, promover mejoras en las educación del alumnado con necesidades educativas especiales y reivindicar las reformas como objetivo básico para la consecución de escuelas de calidad para todas las personas (Echeita y Ainscow, 2010). Así, a partir de finales del siglo XX, en diversos ámbitos y contextos comienza a seguirse una serie de pasos que apuntan a una clara evolución en positivo hacia la educación inclusiva y de calidad (Belmonte Almagro y García Sanz, 2013; Gómez-Vela, 2007).

En la actualidad, se demanda de una escuela inclusiva basada en los principios de equidad, cooperación y solidaridad, que promueva un modelo de enseñanza dirigido por igual a todo el alumnado y centrado en aspectos que fomenten su capacidad de ser autónomos, independientes, autorregulados, y en definitiva, que les permita convertirse en agentes causales de sus propias vidas; esto es, en personas autodeterminadas (Arellano Torres y Peralta López, 2013; Van Loon, 2009; etc.).

La autodeterminación, tomando como referente el Modelo Funcional de Autodeterminación de Wehmeyer (1996, 1998) se refiere a la disposición de cuatro características o elementos: autonomía, autorregulación, capacitación psicológica y autoconocimiento, los cuales evidencian la existencia de complejas relaciones entre la persona y su entorno que se configuran a lo largo de la vida de la persona y cuyo momento crítico es la adolescencia. Es esta una etapa llena de cambios, de nuevos retos y nuevas decisiones, es el momento clave para la puesta en práctica de las capacidades y habilidades adquiridas para tomar decisiones, hacer elecciones, regular las propias acciones, etc. (Carter, Austin y Trainor, 2012; Vega García, Gómez-Vela, Fernández Pulido y Badía Corbella, 2013) por lo que la instrucción directa y la disposición de oportunidades deberían situarse como aspectos centrales en el proceso de aprendizaje del alumnado, de modo que contribuya a aumentar su deseo y sus esfuerzos por alcanzar resultados personales positivos que, finalmente, 
favorezcan el logro de una mayor calidad de vida (Peralta López y González Torres, 2009).

El destacado papel de la autodeterminación como principio y guía de numerosas actuaciones en el campo de la discapacidad, así como la importancia que recibe su promoción como objetivo deseable y posible para todas las personas con discapacidad y, especialmente, para los adolescentes, suscita la necesidad de atender al análisis de estos dos constructos poniendo especial atención al papel de uno de los principales factores ambientales que median en el desarrollo de las habilidades y capacidades conducentes a la adquisición de la conducta autodeterminada, la escuela.

La escuela juega un papel predominante en la capacitación del alumnado con discapacidad para convertirse en dueños y responsables de sus actuaciones. No obstante, en ella se evidencian e ciertas carencias, falta de estabilidad y de continuidad de los apoyos que se le ofrece al alumnado en su proceso de tránsito a la vida adulta (Pallisera et al., 2014).

Por ello, esta investigación tiene por objetivo conocer el nivel de autodeterminación del alumnado con discapacidad intelectual escolarizado en Centros de Educación Ordinaria. En específico se pretende: (a) ofrecer información de tipo descriptivo que refleje los niveles de calidad de vida de este alumnado, atendiendo especialmente al dominio de autodeterminación, (b) estudiar el nivel de autodeterminación del alumnado en base a las cuatro características presentes en este constructo: autonomía, autorregulación, capacitación psicológica y autoconocimiento, y (c) describir los elementos clave que determinan la calidad de la escolarización de este alumnado, reflejando tanto las percepciones positivas como aquellas que son mejorables ofrecidas por el alumnado $y$ por su profesorado.

\section{Método}

Se emplea la metodología de estudio de caso de tipo descriptivo-interpretativo (Yin, 2009) y basada en métodos mixtos para lograr un conocimiento más profundo de la realidad. Se trata ésta de una metodología de creciente uso en investigación sobre calidad de vida (Liesa Orús y Vived Conte, 2010; Pallisera et al., 2014) y sobre autodeterminación (Gregitis, Gelpi, Moore y Dees, 2015; Zacarías Ponce, Saad Dayán, Fiorentini Cañedo y Nava Castro, 2015), útil y coherente con los objetivos de la investigación que versan sobre la necesidad de ofrecer una descripción precisa e intensiva de una realidad muy particular a través del uso de múltiples fuentes de datos.

\section{Participantes}

Se han seleccionado diez estudiantes, de los cuales cinco son hombres y cinco son mujeres, con edades comprendidas entre los 14 y los 16 años, que se encuentran escolarizados en Centros de Educación Ordinaria de la ciudad de A Coruña. Dichos jóvenes presentan discapacidad intelectual leve, moderada o grave, o retraso general del desarrollo (véase Tabla 1).
Tabla 1.

Características de los participantes

\begin{tabular}{|c|c|c|c|}
\hline & & Frecuencia & Porcentaje \\
\hline \multirow[t]{3}{*}{ Género Válidos } & Hombre & 5 & 50.0 \\
\hline & Mujer & 5 & 50.0 \\
\hline & Total & 10 & 100.0 \\
\hline \multirow[t]{4}{*}{ Edad } & 14 & 5 & 50.0 \\
\hline & 15 & 2 & 20.0 \\
\hline & 16 & 3 & 30.0 \\
\hline & Total & 10 & 100.0 \\
\hline \multirow[t]{3}{*}{ Curso } & $1^{\circ} \mathrm{ESO}$ & 6 & 60.0 \\
\hline & $2^{\circ} \mathrm{ESO}$ & 4 & 40.0 \\
\hline & Total & 10 & 100.0 \\
\hline \multirow{7}{*}{$\begin{array}{l}\text { Discapacidad } \\
\text { intelectual }\end{array}$} & Leve & 2 & 20.0 \\
\hline & Moderada & 4 & 40.0 \\
\hline & Grave & 1 & 10.0 \\
\hline & Retraso & & \\
\hline & General del & 3 & 30.0 \\
\hline & Desarrollo & & \\
\hline & Total & 10 & 100.0 \\
\hline
\end{tabular}

El estudio cuenta también con la participación de agentes educativos caracterizados por: (a) permanecer en la institución por tres o más años; y (b) dar atención directa al alumnado encuestado. Son un total de cuatro profesionales: un pedagogo terapéutico (E1), una orientadora con más de treinta años de permanencia en el centro (E2), una orientadora con tres años de permanencia (E3) y una profesora/tutora de un aula de diversificación curricular (E4).

\section{Instrumentos}

Este estudio de casos se realiza a través de técnicas de recogida y análisis de datos cualitativas (análisis documenta y técnica narrativa) y cuantitativas (encuestación). Los instrumentos empleados son:

1. Revisión de la literatura más reciente en materia de calidad de vida y autodeterminación, útil para fundamentar el estudio, las áreas de interés en la investigación, y contrastar la información obtenida durante el trabajo de campo.

2. Aplicación de dos cuestionarios: (a) Cuestionario de Evaluación de la Autodeterminación (Verdugo et al., 2014), un instrumento compuesto por 61 ítems que recogen información acerca de los cuatro componentes que conforman la conducta autodeterminada, en base al Modelo Funcional de Wehmeyer (1996, 1999); (b) el Cuestionario de Evaluación de la Calidad de Vida de Alumnos Adolescentes (CCVA, Gómez-Vela y Verdugo, 2009), compuesto por 61 ítems, de los cuales siete se orientan a controlar la aquiescencia y otros diez el sesgo derivado de la deseabilidad social.

3. Entrevistas semi-estructuradas con los profesionales de los centros, en las que se preguntaba acerca de cuatro bloques de contenidos: (a) autodeterminación y desarrollo personal; (b) relaciones interpersonales, 
inclusión y derechos; (c) bienestar físico, emocional y material; y (d) autodeterminación y calidad de vida.

4. Entrevistas al alumnado, por medio de las diez preguntas formuladas en la sección cualitativa del CCVA acerca de diversas áreas de su vida.

\section{Procedimiento}

La recogida de datos se llevó a cabo a lo largo de los meses de enero y marzo de 2015. En primer lugar, se tomó contacto con varios centros educativos de la ciudad por medio de una carta y, posteriormente, una llamada telefónica. En la primera fase del estudio se obtuvo respuesta de seis centros, de los que seleccionamos aquellos en los que obtuvimos además la participación del profesorado.

Una vez obtenida la información, los datos fueron analizados estadísticamente, utilizando los relatos del profesorado y del alumnado como información que fundamenta y contextualiza los resultados. Para el análisis de los cuestionarios se empleó el paquete estadístico IBM SPSS Statistics 20. Para el análisis del primer cuestionario, se agruparon los ítems contenidos en el CCVA en siete dominios correspondientes a las dimensiones conceptualizadas en el Modelo de Calidad de Vida en la adolescencia de Gómez-Vela y Verdugo (2009). En análisis del segundo cuestionario se produjo mediante la agrupación de los ítems en cuatro factores, que coinciden con las secciones formuladas en el Modelo de Autodeterminación Funcional de Wehmeyer (1996, 1998), anteriormente citados.

El procedimiento para el análisis de la información cualitativa se dividió en dos fases: (a) fase descriptiva, en la que se codificó la información de mayor relevancia y se establecieron los códigos principales del estudio que fueron contrastados con la información contenida en la revisión bibliográfica inicial; y (b) fase interpretativa, basada en la comprensión de las relaciones entre códigos o variables y la extracción de las principales categorías de cada caso, evidenciando también la influencia de variables como el sexo, la edad, el interés y la motivación del alumnado, las percepciones y las expectativas del profesorado hacia éstos, la percepción de la relación familia-escuela por parte del profesorado, etc.

Este proceso sistemático de recogida y análisis de la información procedente de diversas fuentes y agentes, y a través de diferentes técnicas, ha facilitado la triangulación de la información así como generar un conocimiento más profundo de la realidad de estos jóvenes, disminuyendo así el influjo de problemas relacionados con la validez y la fiabilidad derivados del posible sesgo de la investigadora.

\section{Resultados}

Se presentan los principales hallazgos obtenidos del análisis de los cuestionarios aplicados al alumnado en relación con la calidad de vida (véase Tabla 2) y autodeterminación (véase Figura 1) y las entrevistas a los profesionales de los centros participantes.

En la Tabla 2 se muestran los resultados obtenidos por el alumnado participante en cada dimensión de la calidad de vida, estimados en una escala 0-100.
Tabla 2.

Estadísticos descriptivos de las puntuaciones transformadas de la calidad de vida

\begin{tabular}{lccc}
\hline & N & Media & Desv. Tip \\
\hline Relaciones interpersonales & 10 & 93.5 & 37.899 \\
Bienestar material & 10 & 62.5 & 17.024 \\
Desarrollo personal & 10 & 81.07 & 10.247 \\
Bienestar emocional & 10 & 82.85 & 12.463 \\
Integración/presencia en la & 10 & 62.70 & 12.323 \\
comunidad & 10 & 69 & 8.755 \\
Bienestar físico & 10 & 64.64 & 10.839 \\
Autodeterminación & & & \\
\hline
\end{tabular}

En este sentido, se observan valores que oscilan entre el 62 y el 93\% aproximadamente. Se trata de valores medios, teniendo en cuenta la decisión de los autores de la escala de situar en el 70\% un nivel "satisfactorio" de Calidad de Vida (Gómez-Vela y Verdugo, 2009).

\section{Análisis de las dimensiones de la calidad de vida}

El análisis de la información correspondiente a cada dimensión de la calidad de vida de estos jóvenes permite destacar, en términos generales, una valoración muy positiva de los dominios de relaciones interpersonales (93.5\%), bienestar emocional (82.85\%) y desarrollo personal (81.07\%), y puntuaciones bajas en relación con bienestar físico (69\%), autodeterminación (64.64\%), integración en la comunidad (62.7\%) y bienestar material (62.5\%).

Dichas puntuaciones presentan notables variaciones en cada joven, por lo que a continuación analiza exhaustivamente los siete dominios que componen la calidad de vida, agrupados en tres temáticas: bienestar personal, participación social e independencia personal.

Bienestar personal. Se recoge, bajo este epígrafe, las dimensiones de la calidad de vida que aluden al bienestar de la persona en términos generales; a saber, bienestar emocional, físico y material. Respecto al primero, los resultados refieren un bienestar emocional de estos jóvenes en términos medios, que se expresa además en sus definiciones de la felicidad como "estar al lado de mi hermana que me quiere" (A1), "estar con mis amigos" (A5), "estar contenta y tener alegría” (A7), “estar contento” (A8), etc. Esta dimensión está representada por un promedio de $82.85 \%$. Estos jóvenes perciben su bienestar emocional condicionado por el apoyo y el cariño recibido por sus familias, sus vivencias en el instituto y su relación con sus compañeros. Al respecto, el profesorado entrevistado se refiere a una buena relación entre este alumnado y sus familias que, en ocasiones, se caracteriza por la "sobreprotección”. Con respecto a sus compañeros, la mayoría de profesionales afirman que este alumnado es aceptado y apreciado por sus compañeros, aunque no siempre apoyado ya que "Para apoyar habría que saber cómo y, también, coincidir en los intereses y esto, en muchas ocasiones, no ocurre" (E1) y, por lo tanto, 
aprecia, acepta, no apoya. Eeh, admite en el 90\% de los casos. Hay quien se mofa, como lo hay siempre, hay quien se mete con ellos, lo de siempre, pero yo creo que:: bueno, lo bueno que ha tenido la integración es que:: ya vienen acostumbrados a ellos desde siempre, vienen acostumbrados a que exista la diversidad entre ellos desde educación infantil, entonces, aceptan que están ahí, y los admiten. (E2)

Respecto al bienestar físico, las valoraciones de este alumnado son, a nivel general, altas. La mayoría de ellos se refieren a un buen estado de salud. De forma espontánea, se refieren a la salud como comer sano, hacer deporte dentro o fuera de la escuela, etc.

Por último, el bienestar material obtiene valoraciones $62.5 \%$ Se observa que las puntuaciones más bajas se relacionan no tanto con la disposición de bienes básicos (alimento, vivienda, vestido), sino más bien con las posibilidades que sus padres les ofrecen para gestionar y administrar su propio dinero para comprarse cosas o para ahorrar.

Participación social. Se incluyen en este apartado los dominios de relaciones interpersonales e integración y presencia en la comunidad. Las relaciones interpersonales obtienen una puntuación de 93.5\% Anteriormente, se evidenciaba la importancia que para estos jóvenes tiene contar con apoyo familiar. Las familias, en opinión de las profesionales de los centros, son un eje clave en la educación del alumnado, tanto como facilitadoras como barreras a su desarrollo:

Tú, como padre haces el esfuerzo de::: trabajar por Tú hijo, entonces ahí estás ya reconociendo sus potencialidades porque se las aceptas, aceptas que las tiene porque estás TÚ esforzándote por ello. Si no haces ese esfuerzo no le estás::: reconociendo nada, le estás haciendo dependiente y entonces en este sentido, pues sí, hay familias que efectivamente están implicadas en la evolución de sus hijos (E3)

Y en relación con esto, la integración de estos jóvenes se verá en gran medida determinada también por el apoyo familiar recibido pues la familia actúa como promotora/barrera a la inclusión de sus hijos, al igual que la sociedad. Opina uno de los profesionales que el mundo "está diseñado por personas sin discapacidad para personas sin discapacidad" (E1) limitando por tanto su acceso y en consecuencia, la persona con discapacidad "se percibe como una persona, o sea, nula” (E4).

Tampoco la escuela está actuando como facilitadora de la integración del alumnado. Se reclama del contexto educativo que actúa como "un agente inclusivo" con la sociedad en su conjunto puesto que "la salida laboral de las PCD más que un problema educativo es un problema social y en España se hace muy poco en este sentido (...) las PCD están abocadas a la exclusión social” (E1)

Concuerdan estas afirmaciones con las valoraciones ofrecidas por los docentes acerca de la situación actual que vive el alumnado en relación a sus posibilidades para participar en la sociedad, donde frente a una valoración muy positiva del apoyo recibido por sus redes más cercanas (las familias), la percepción de su integración en la sociedad es más de treinta puntos por debajo.
Independencia personal. Se refiere a las dimensiones de desarrollo personal y autodeterminación. Respecto al primero, en general estos jóvenes afirman que sus logros son el resultado de sus esfuerzos, teniendo presentes también sus dificultades académicas. En su mayoría, desconocen la finalidad real de su formación en el futuro, aspecto reforzado en cierta medida por los profesionales que trabajan con ellos, cuyas expectativas respecto a este alumnado son, generalmente, bajas: "Estos niños si es sin una adaptación, una ACI, y sin un programa de diversificación curricular directamente no se llevarían un título. Aún así, el título es un poco regalado, porque:: pero bueno, es una manera de regalárselo” (E4)

Finalmente, en lo referente a la autodeterminación de estos jóvenes, el trazo común que evidencia el profesorado es la "sobreprotección”. Como afirma una de las profesionales

Tenemos tendencia a superprotegerlos, a no dejar que sean lo más autónomos posibles [mm]. Yo estoy segura de que muchos de los que vienen aquí o:: la mitad, por lo menos, podrían ser más autónomos si no estuvieran tan pendientes de ellos (E2)

Esta sobreprotección coincide con la bajas puntuaciones en este dominio de la calidad de vida (64.64\%), así como en la escala ARC-INICO, donde se obtiene un promedio de $66.84 \%$, y en las diferentes secciones que lo componen, sobre todo en referencia a su autorregulación (63.95\%) y su autonomía (64.7\%) (véase Figura 1).

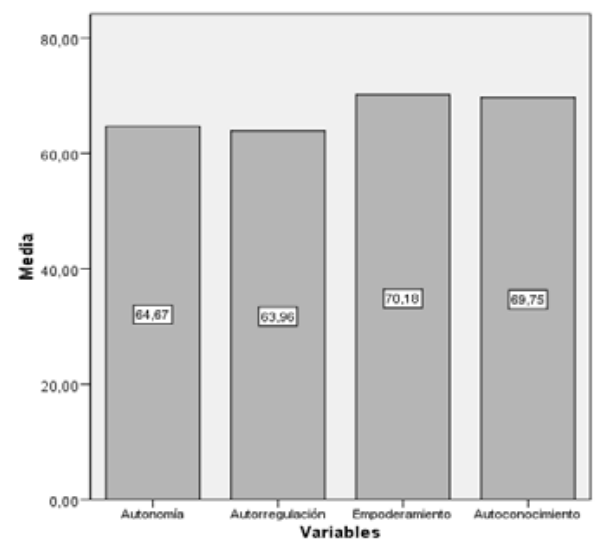

Figura 1. Puntuaciones medias en las secciones de la autodeterminación.

Los resultados obtenidos ponen de manifiesto que estos adolescentes continúan viendo limitadas sus capacidades para tomar decisiones, hacer elecciones, tomar el control en aspectos relacionados con su educación, sus amistades, su tiempo de ocio, etc.

\section{Discusión}

Los resultados parecen vislumbrar la relación entre una mayor calidad de vida y autodeterminación y las variables contrastadas, siendo necesario intervenir específicamente para la promoción de las habilidades y capacidades que les permitan alcanzar una mayor autodeterminación y, por consiguiente, contribuir al incremento de su calidad de vida.

Si bien comienzan a apreciarse, en muchos de estos jóvenes, el desarrollo de habilidades y capacidades que 
indican una progresión hacia un estado de independencia, autonomía y toma de control sobre sus vidas, es fundamental el trabajo continuo y progresivo, pues estos jóvenes se encuentran en un momento de su vida lleno de cambios, de retos y de dificultades a los que deberán enfrentarse. Si los docentes y las familias les proporcionan los recursos y estrategias adecuadas, y si crean un entorno adecuado, será posible que estos jóvenes avancen hacia el camino de la autodeterminación. Si por el contrario, creamos barreras, los sobreprotegemos y no les damos esos entornos naturales y enriquecedores para poner a prueba sus capacidades y descubrir sus limitaciones y cómo afrontarlas, estaremos creando seres dependientes y apáticos, esclavos de sus propias limitaciones.

En este grupo, se atisban comportamientos y percepciones de la vida muy diferentes y por ello, debemos atender a sus individualidades. Ellos deben sentirse apoyados en todo momento partiendo de sus necesidades, de sus capacidades y potencialidades y reconociendo y ayudándoles a superar sus limitaciones.

Por todo ello se hace fundamental el papel de la escuela, del profesor/a-tutor/a, y de su coordinación con cada familia, para fomentar la adquisición de las habilidades que cada joven precisa, dotando a cada cual y al conjunto de los estímulos y de las oportunidades que facilitarán que, todos aquellos aprendizajes de los que los hemos provisto, puedan ser expresados en las situaciones lo más naturales y reales posibles, permitiéndoles adquirir mayores experiencias y conocimientos en autodeterminación.

\section{Referencias}

Arellano Torres, A. y Peralta López, F. (2013). Autodeterminación de las personas con discapacidad intelectual como objetivo educativo y derecho básico: estado de la cuestión. Revista Española de Discapacidad, $\quad$ 1(1), $97117 . \quad$ doi.: 10.5569/2340-5104.01.01.05

Carter, E., Austin, D. y Trainor, A.A. (2012). Predictors of postschool employment outcomes for Young adults with severe disabilities. Journal of Disability Policy Studies, 23(1), 50-63. doi.: 10.1177/1044207311414680

Echeita, G. y Ainscow, M. (2010). La educación inclusiva como derecho. Marco de referencia y pautas de acción para el desarrollo de una revolución pendiente. Trabajo presentado en el II Congreso Iberoamericano de Síndrome de Down (Down España, Granada).

Gómez-Vela, M. y Verdugo, M.A. (2009). Cuestionario de evaluación de la calidad de vida en alumnos adolescentes (CCVA).Madrid: CEPE.

Gregitis, S., Gelpi, T., Moore, B. \& Dees, M. (2015). Self-determination skills of adolescents enrolled in special education: an analysis of four cases. Occupational Therapy in Mental Health, 26(1), 67-84. http://dx.doi.org/10.1080/01642120802647683

Liesa Orús, M. y Vived Conte, E. (2010). Discapacidad, edad adulta y vida independiente. Un estudio de casos. Educación y Diversidad, 4(1), 101-124. de http://sid.usal.es/articulos/discapacidad/15188/8-26/discapacidad-edad-adulta-y-vida-independiente-unestudio-de-casos.aspx

Pallisera, M., Fullana, J., Vilá, M, Jiménez, P., Castro, M., Puyalto... Martin, R. (2014). Análisis de los apoyos que reciben los jóvenes con discapacidad intelectual en su transición a la vida adulta en España: una investigación a partir de experiencias profesionales y personas con discapacidad. Revista Española de Discapacidad, 2(2), 27-44. doi.:10.5569/2340-5104.02.02.02

Peralta López, F. y González-Torres, C. (2009). El movimiento hacia la autodeterminación personal: antecedentes y estado actual. Trabajo presentado en el XV Coloquio de Historia de la Educación: El largo camino hacia la una educación inclusiva: la educación especial y social del siglo XIX hasta nuestros días (pp. 181-192). Pamplona: Universidad Pública de Navarra.

Schalock, R.L., Gardner, J.F. y Bradley, V.J. (2009). Quality of life for people with intelectual and other development disabilities. Applications across individuals, organizations, communities and systems ( $2^{\circ}$ ed). Washington, DC: American Association on Intelectual and Developmental Disabilities.

Vega García, C., Gómez Vela, M., Fernández Pulido, R. y Badía Corbella, M. (2013). El papel del contexto educativo en la autodeterminación. Análisis de su influencia en el proceso de transición a la vida adulta de alumnos con discapacidad intelectual. Revista Iberoamericana de Educación, 63, 19-33. http://riberdis.cedd.net/bitstream/handle/1118 1/4359/El_papel_del_contexto_educativo.pdf?sequen $\mathrm{ce}=1 \& \mathrm{rd}=0031371311959578$

Verdugo Alonso, M.A., Vicente Sánchez, E., Gómez-Vela, M., Fernández Pulido, R., Wehmeyer, M.L., Badía Corbella, M.... Calvo Álvarez, M.I. (2014). Escala ARC-INICO de evaluación de la autodeterminación. Manual de aplicación y corrección. Salamanca: Publicaciones del INICO.

Wehmeyer, M.L. (1996). Self-determination as an educational outcome: Why is it important to children, youth and adults with disabilities?. En D.J. Sands y M.L. Wehmeyer (Eds.), Self-determination across the life span: independence and choice for people with disabilities (pp. 15-34). Baltimore: Paul H. Brookes.

(1998). A functional model of self-determination: describing development and implementing instruction. Focus on Autism and Other Developmental Disabilities, 14(1), 53-61. http://www.imdetermined.org/files_images/gen eral/SD5_A\%20Functional\%20Model\%20of.pdf

Yin, R.K. (2009). Case study research: design and methods (4th ed., Vol. 5). Londres, Reino Unido: Sage.

Zacarías Ponce, J., Saad Dayán, E., Fiorentini Cañedo, Z. \& Nava Castro, E. (2015). Desarrollo de la autodeterminación en jóvenes con discapacidad intelectual que participan en un entorno universitario. Actas de las IX Jornadas Científicas Internacionales de Investigación sobre Personas con Discapacidad (pp. 1-11). Salamanca: INICO. http://cdjornadas-inico.usal.es/docs/332.pdf 